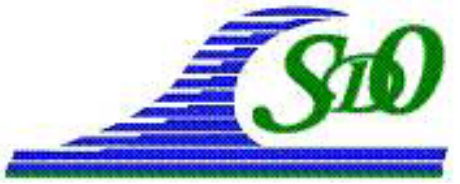

$X^{\text {èmes }}$ Journées Nationales Génie Côtier - Génie Civil

Les Sables d'Olonne, 22-25 juin 2010

DOI:10.5150/jngcgc.2010.023-C C Editions Paralia CFL

disponible en ligne - http://www.paralia.fr - available online

\title{
Evaluation des flux sédimentaires sur les plages macrotidales du Nord-Pas-de-Calais à partir de données hydrodynamiques et de piégeages in situ
}

\author{
Adrien CARTIER ${ }^{1}$, Arnaud HEQUETTE ${ }^{1}$
}

\section{Laboratoire d'Océanologie et de Géosciences, UMR CNRS 8187 LOG, Université du Littoral Côte d'Opale 189A Avenue Maurice Schumann, 59140 Dunkerque. France. \\ adrien.cartier@univ-littoral.fr ; arnaud.hequette@univ-littoral.fr}

\section{Résumé :}

Les transports sédimentaires longshore ont été mesurés sur trois plages macrotidales à barres et à bâches du Nord-Pas-de-Calais. Les flux sédimentaires ont été évalués à l'aide de pièges à sédiments suivant la méthode proposée par KRAUS (1987) et ont été associés à des mesures hydrodynamiques réalisées sur l'estran. Nos résultats montrent une bonne corrélation des flux avec la vitesse moyenne du courant et une plus faible relation avec la hauteur significative de la houle. Lors de conditions de faible agitation, les courants de marée se sont avérés peu efficaces pour induire un transport sédimentaire important, le rôle des vagues semblant déterminant pour la mise en mouvement des sédiments. Nos résultats montrent également que la variation longitudinale du transport sédimentaire longshore est faible, mais que les flux peuvent augmenter localement en fonction de la morphologie de l'estran, notamment à proximité des chenaux de vidange des bâches.

\section{Mots clés :}

Flux sédimentaires - Plages macrotidales - Piégeage in situ - Nord-Pas-de-Calais

\begin{abstract}
:
Longshore sediment transport was measured on three macrotidal barred beaches of "Nord-Pas-de-Calais" (Northern France). Sediment fluxes were estimated using streamer traps, following the method proposed by KRAUS (1987), and compared with hydrodynamic data obtained across the beach. Our results show a good correlation between sediment fluxes and current velocities, but a weaker relationship with significant wave heights. During conditions of low wave activity, tidal currents were only responsible for low sediment transport rates, showing that waves play a major role in the initiation of sediment motion. Our results also show limited longshore variation in longshore transport, although sediment transport rate can locally increase in the vicinity of intertidal drainage channels associated with bar-trough beach topography.
\end{abstract}

Key words:

Sediment flux - Macrotidal beach - Streamer trap - Nord-Pas-de-Calais - France 


\section{Introduction}

L'évaluation des flux sédimentaires sur les plages est un exercice complexe en raison de la forte variabilité des nombreux paramètres liés à la houle et aux courants, dont dépendent les transports particulaires dans la zone intertidale (BAYRAM et al., 2007). Cette complexité est particulièrement grande en milieu macrotidal en raison de l'influence des courants tidaux qui se combinent aux courants oscillatoires induits par les vagues, ainsi qu'en raison des fluctuations du niveau d'eau dues à la marée. Bien que quelques études aient été consacrées à l'évaluation des transports sédimentaires dans les environnements côtiers macrotidaux, elles sont généralement basées sur l'utilisation de traceurs fluorescents (LEVOY et al., 2000 ; VOULGARIS et al., 1998) ou consistent en des estimations des transports sédimentaires potentiels reposant sur des modèles numériques (SEDRATI \& ANTHONY, 2007 ). Le travail présenté ici repose sur une combinaison de mesures hydrodynamiques à haute fréquence et de mesures simultanées des flux sédimentaires réalisées à l'aide de pièges à sédiments sur des plages du Nord-Pas-de-Calais soumises à un marnage macrotidal.

\section{Zones d'études}

Les sites d'études correspondent à trois plages sableuses à barres intertidales de la côte du Nord-Pas-de-Calais (Nord de la France) situées à Zuydcoote, Wissant et Hardelot Plage (figure 1).

Le site de Zuydcoote, situé à l'est de Dunkerque, est caractérisé par une plage de sables fins $\left(\mathrm{D}_{50}=0,2 \mathrm{~mm}\right)$, de 350 à $400 \mathrm{~m}$ de largeur, ayant une pente moyenne d'environ $0,014(\tan \beta)$. L'amplitude de la marée varie de $3,4 \mathrm{~m}$ en mortes-eaux à $5,2 \mathrm{~m}$ en viveseaux moyennes, et ce site peut par conséquent être considéré comme méso à macrotidal. La côte, orientée NE-SO, fait face à la mer du Nord, caractérisée par une houle courte à fetch limité. Le site de Wissant est situé dans une baie qui s'étend sur plus de $6 \mathrm{~km}$, limité au sud par le Cap Gris Nez et au Nord par le Cap Blanc Nez. De par sa situation géographique, la baie de Wissant est caractérisée par un hydrodynamisme plus puissant en raison de sa localisation sur la rive du détroit du Nord-Pas-de-Calais où les échanges de masse d'eau entre la mer du Nord et la Manche sont particulièrement intenses. Le site de mesures est situé dans la partie est de la baie, caractérisée par une plage de sables fins $\left(D_{50}=0,22 \mathrm{~mm}\right)$ qui adopte une pente moyenne de 0,012 . La côte est soumise à un marnage allant de 4,2 $\mathrm{m}$ à $6,7 \mathrm{~m}$ pour les marées de vives eaux et mortes eaux moyennes. Le troisième site est situé à Hardelot Plage, au niveau de la dune du Mont St-Frieux. La plage est composée de sables fins $\left(\mathrm{D}_{50}=0,23 \mathrm{~mm}\right)$ et possède une pente moyenne de 0,026. L'amplitude de la marée atteint 4,8 $\mathrm{m}$ en morte eau moyenne et 8,0 $\mathrm{m}$ en période de vive eau. Orientée N-S, la côte fait face à la Manche.

Les courants de marée sur ces trois plages s'écoulent parallèlement au rivage et sont caractérisés par une dissymétrie responsable d'une dominance des courants de flot par rapport aux courants de jusant. Cette dominance de courants de flot, combinée à un 
régime de vents et de houles en provenance du SO, génère une circulation hydrodynamique et des transports sédimentaires dirigés vers l'est sur le littoral de la mer du Nord et vers le nord sur le rivage de la Manche (SIPKA \& ANTHONY, 1999).

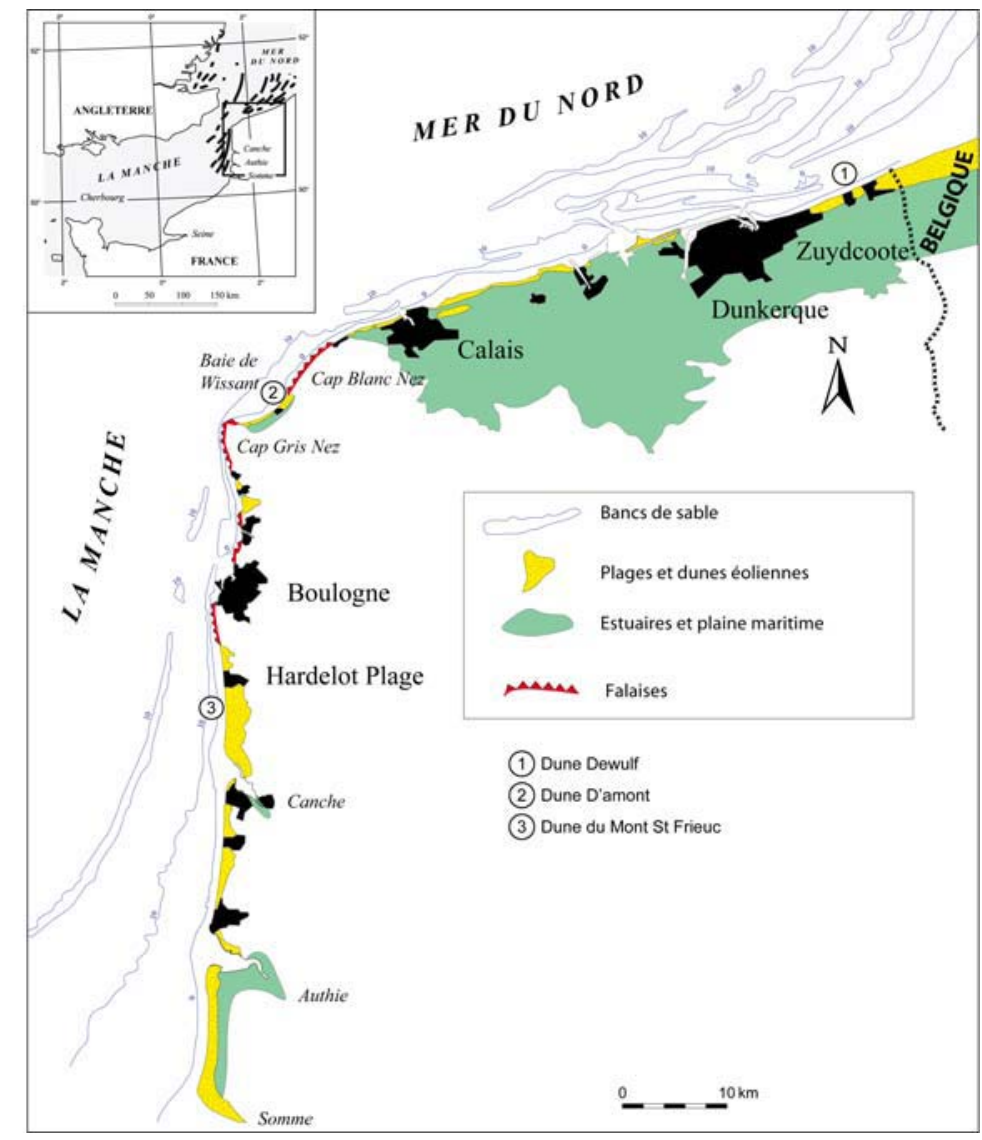

Figure 1. Situation géographique des sites d'études

(Modifiée d'après ANTHONY \& HEQUETTE, 2007).

\section{Matériels et méthodes}

La méthode utilisée repose sur l'acquisition simultanée de données courantométriques et de mesures des flux sédimentaires à l'aide de pièges à sédiments. Les paramètres hydrodynamiques ont été mesurés à l'aide de quatre appareils courantométriques : deux courantomètres-houlographes électromagnétiques (Valeport) et deux profileurs de courant (ADCP). Ces appareils permettant d'obtenir des mesures des paramètres de houle (hauteur, période et direction) ainsi que la vitesse et la direction des courants, ont été disposés sur les barres intertidales en milieu et bas des plages le long de deux radiales espacées de 100 mètres (figure 2).

Les mesures des flux sédimentaires ont été réalisées en utilisant des pièges à sédiments de type KRAUS (1987) qui permettent de mesurer le transport sédimentaire en suspension à plusieurs niveaux dans la colonne d'eau et dans la couche de fond grâce à cinq filets d'une maille de $63 \mu \mathrm{m}$ répartis régulièrement sur une hauteur de $1,43 \mathrm{~m}$. Ces 
mesures ont été effectuées au voisinage direct des appareils courantométriques afin de pouvoir comparer les flux calculés avec les différents forçages hydrodynamiques. Les piégeages ont été effectués à différentes positions le long de chaque radiale, dix piégeages étant réalisés lors de chaque marée : cinq lors du flot et cinq pendant le jusant (figure 2).

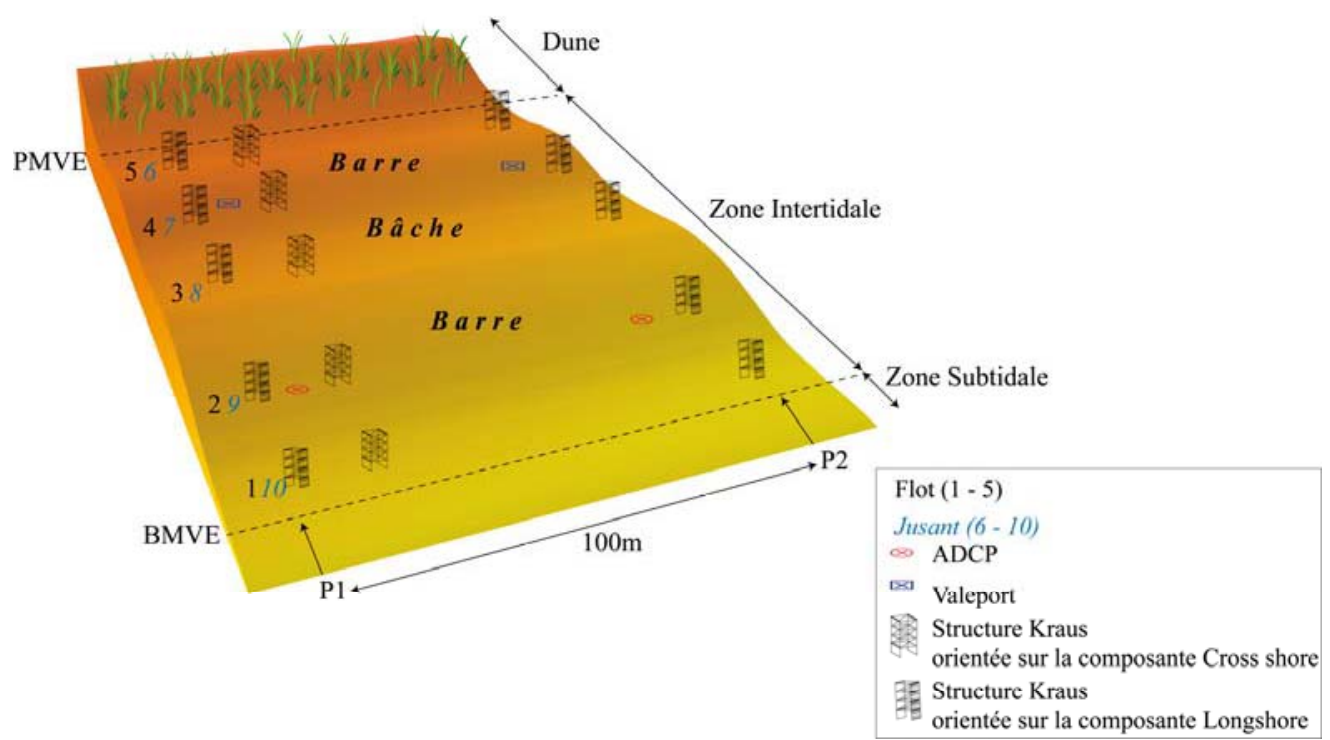

Figure 2. Positionnement des appareils de mesures courantométriques et des différents pièges à sédiment de type KRAUS (1987).

Lors de chaque opération de piégeage, deux structures étaient placées face au courant longitudinal et une troisième face à la houle pendant une durée de 10 minutes. Les sédiments recueillis ont été ensuite rincés à l'eau douce puis passés sur un tamis de $63 \mu \mathrm{m}$ afin d'éliminer la fraction fine. Les échantillons ont enfin été séchés en étuve puis pesés. Un flux est déterminé pour chaque niveau d'échantillonnage au-dessus du fond, puis intégré sur la colonne d'eau en suivant la procédure préconisée par ROSATI \& KRAUS (1989) :

$F=h \sum_{i=1}^{N} F(i)+\sum_{i=1}^{N-1} a(i) * F E(i)$

où $F$ est le flux intégré sur la colonne d'eau $\left(\mathrm{kg} \mathrm{s}^{-1} \mathrm{~m}^{-1}\right), h$ est la hauteur de l'ouverture du piège $(\mathrm{m}), F(i)$ est le flux mesuré au niveau de chacun des pièges $\left(\mathrm{kg} \mathrm{s}^{-1} \mathrm{~m}^{-2}\right), a(i)$ est la distance entre chaque piège $(\mathrm{m}), F E(i)$ est le flux intermédiaire $\left(\mathrm{kg} \mathrm{s}^{-1} \mathrm{~m}^{-2}\right)$ et $\mathrm{N}=5$.

\section{Résultats}

Le tableau 1 résume les conditions hydrodynamiques enregistrées par les 4 appareils courantométriques lors des différentes campagnes de terrain. L'intensité des courants et les conditions d'agitations présentent une grande variabilité d'une campagne à l'autre. Les conditions ont pu être extrêmement calmes, comme à Hardelot en mai-juin 2009 où 
les vitesses de courants n'ont atteint que $0,15 \mathrm{~m} \mathrm{~s}^{-1}$ avec des hauteurs de vagues maximales de $0,31 \mathrm{~m}$, ou au contraire beaucoup plus énergétique comme en mai 2009 à Wissant alors que les vitesses de courant ont atteint $1,1 \mathrm{~m} \mathrm{~s}^{-1}$ et que les hauteurs maximum des vagues ont été de $0,7 \mathrm{~m}$. Lors de la campagne de Zuydcoote, la plage a été soumise à un hydrodynamisme modéré avec des vitesses de courant maximum de $0,37 \mathrm{~m} \mathrm{~s}^{-1}$ et des vagues de $0,35 \mathrm{~m}$. La direction de la houle a peu varié au cours de chaque campagne. Au niveau de Zuydcoote, les houles étaient orientées NNE à NE. A Wissant, la houle était orienté ONO à OSO avec cependant de rares houles venant du $\mathrm{NE}$, le 13 et 14 mars 2009. Enfin, lors de la campagne d'Hardelot, les houles étaient de secteur OSO à ONO.

Tableau 1. Caractéristiques hydrodynamiques minimales et maximales durant les campagnes de terrains. Paramètres calculés pendant les phases d'échantillonnages au voisinage des appareils : Hs : Hauteur significative ; Tp : Période pic ; V: Vitesse du courant; FL : Flux longshore ; FC : Flux cross shore ; $D$ : Fréquence directionnelle du transport sédimentaire.

\begin{tabular}{|c|c|c|c|c|c|c|c|c|c|}
\hline Campagnes & & $\begin{array}{l}\text { Marnage } \\
(\mathrm{m})\end{array}$ & Coeff. & $\begin{array}{l}H_{s} \\
(m) \\
\end{array}$ & $\begin{array}{l}T_{p} \\
(s)\end{array}$ & $\begin{array}{l}V \\
\left(m s^{-1}\right) \\
\end{array}$ & $\begin{array}{l}F L \\
\left(10^{-3} \mathrm{~kg} \mathrm{~s}^{-1} \mathrm{~m}^{-1}\right) \\
\end{array}$ & $\begin{array}{l}F C \\
\left(10^{-3} \mathrm{~kg} \mathrm{~s}^{-1} \mathrm{~m}^{-1}\right)\end{array}$ & $D$ \\
\hline Zuydcoote & Min & 4,93 & 77 & 0,10 & 4 & 0,02 & 0,02 & 0,77 & \multirow{2}{*}{$100 \% E$} \\
\hline$(13 / 11 / 08$ aи $17 / 11 / 08)$ & $\operatorname{Max}$ & 5,6 & 100 & 0,35 & 10 & 0,37 & 0,49 & 1,9 & \\
\hline Wissant & Min & 4,09 & 71 & 0,09 & 4 & 0,03 & 0,02 & - & \multirow{2}{*}{$81 \% E$} \\
\hline$(07 / 03 / 09$ au $14 / 03 / 09)$ & $\operatorname{Max}$ & 7,35 & 108 & 0,69 & 11 & 1,1 & 14,24 & - & \\
\hline Hardelot & Min & 5,61 & 57 & 0,11 & 4 & 0,09 & 0,009 & 0,05 & \multirow{2}{*}{$54 \% S$} \\
\hline$(30 / 05 / 09$ аи 06/06/09) & $\operatorname{Max}$ & 6,98 & 71 & 0,31 & 9 & 0,15 & 0,18 & 0,53 & \\
\hline
\end{tabular}

En raison de cette variabilité dans les conditions hydrodynamiques, les flux sédimentaires mesurés ont également été caractérisés par une forte variabilité d'un site à l'autre. Lors de conditions de faible énergie, comme en mai-juin 2009 à Hardelot, les flux longshore maximum n'ont atteint que $0,18 \times 10^{-3} \mathrm{~kg} \mathrm{~s}^{-1} \mathrm{~m}^{-1}$. A Zuydcoote, à la faveur de conditions hydrodynamiques plus fortes, notamment en terme de vitesse de courant, nos mesures ont permis de mettre en évidence un transport sédimentaire plus élevé, le transport longshore atteignant une valeur maximale de $0,49 \times 10^{-3} \mathrm{~kg} \mathrm{~s}^{-1} \mathrm{~m}^{-1}$. C'est enfin à Wissant que les flux longshore les plus importants ont été mesurés, dépassant $14 \times 10^{-3} \mathrm{~kg} \mathrm{~s}^{-1} \mathrm{~m}^{-1}$ lors de conditions de forte agitation.

Les données recueillies lors de la campagne de Wissant montrent une relation puissance des flux sédimentaires avec la hauteur significative de la houle et la vitesse moyenne du courant mesurée à 0,20 et $0,40 \mathrm{~m}$ au dessus du fond (figure 3a). La corrélation est toutefois meilleure avec la vitesse du courant puisque les coefficients de régression $\left(\mathrm{R}^{2}\right)$ atteignent $0,47(\mathrm{n}=10)$ pour les vitesses à $0,20 \mathrm{~m}$ et $0,56(\mathrm{n}=12)$ à $0,40 \mathrm{~m}$, alors qu'elle est seulement de $0,29(\mathrm{n}=22)$ avec la hauteur de la houle. Les données recueillies à Hardelot (mai-juin, 2009) présentent des relations moins nettes puisque la corrélation des flux avec les vitesses ne s'élève qu'à 0,11 à $0,20 \mathrm{~m}$, mais elle atteint $0,74(\mathrm{n}=10)$ à 
0,40 m (figure 3b). A Zuydcoote, le faible nombre d'échantillonnages au voisinage des appareils ne permet pas de mettre en évidence de relations.

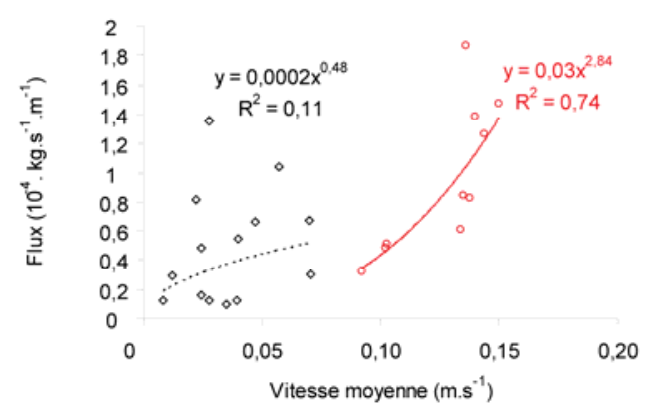

(a)

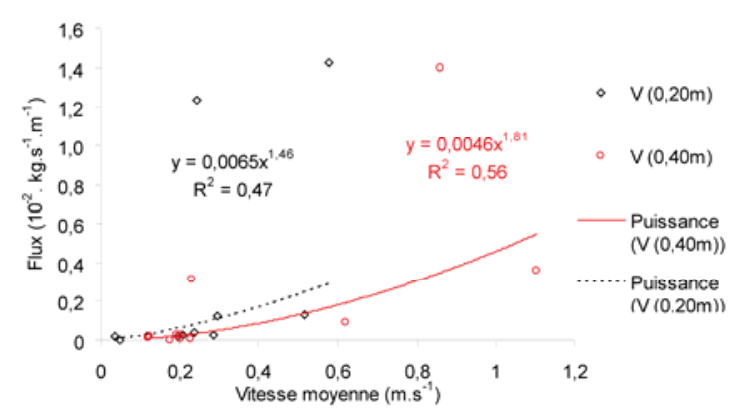

(b)

Figure 3. Corrélation des flux sédimentaires calculés à partir de piégeage in situ avec les vitesses de courant moyens à 0,20 et 0,40 $\mathrm{m}$ au dessus du fond ( $a$ : Hardelot ; $b$ : Wissant).

Les résultats obtenus lors de ces différentes campagnes de mesures montrent que plus les vitesses de courant sont élevées plus la relation avec le transport sédimentaire est bonne. Lorsque les conditions d'agitation sont faibles, il est probable que la marge d'erreur inhérente à la méthode d'échantillonnage soit trop importante pour que de faibles variations de flux sédimentaires puissent être mesurées. Par exemple à Hardelot (mai-juin 2009) lorsque les vitesses sont très faibles $\left(<0,08 \mathrm{~m} \mathrm{~s}^{-1}\right)$, la corrélation avec les flux est inexistante alors qu'une bonne relation est visible pour des vitesses plus élevées. Le piégeage Kraus nécessite des conditions d'énergie minimales afin d'obtenir des résultats suffisamment précis pour être significatifs.

La figure 4 présente la comparaison des flux sédimentaires longshore mesurés simultanément au niveau des deux radiales d'échantillonnage (P1 et P2) lors de chaque campagne (sauf Zuydcoote, novembre 2009). Lorsque l'on compare les flux sédimentaires au niveau du profil P1 avec ceux qui ont été mesurés au niveau du profil P2 en baie de Wissant, on obtient une excellente corrélation $\left(\mathrm{R}^{2}=0,94 ; \mathrm{n}=23\right)$, ce qui montre que l'intensité du transport sédimentaire longshore semble peu varier le long de la plage. Cependant, ce graphique ne prend pas en compte les échantillons prélevés le 9 mars 2009, car la relation serait alors seulement de $0,36(n=24)$. Un des piégeages effectués le 9 mars au cours du jusant était positionné à proximité d'un chenal de vidange, ce qui a pu causer localement une augmentation du flux sédimentaire (figure 5).

Les analyses réalisées avec les données recueillies pendant les campagnes de Zuydcoote en novembre 2008 et Hardelot en mai-juin 2009 présentent des relations similaires. La variabilité des flux sédimentaires a cependant été plus importante à Zuydcoote $\left(\mathrm{R}^{2}=0,42\right)$, mais ceci semble être dû au faible nombre d'échantillons $(\mathrm{n}=8)$. En revanche, une corrélation de $0,85(\mathrm{n}=27)$ a été obtenue pour les données recueillies lors de la 
campagne d'Hardelot, et ce malgré des conditions d'énergie extrêmement faibles (figure 4). Il semblerait donc que la variation longitudinale du transport sédimentaire longshore soit faible, mais que les flux puissent augmenter localement en fonction de la morphologie de l'estran, notamment à proximité des chenaux de vidange des bâches.

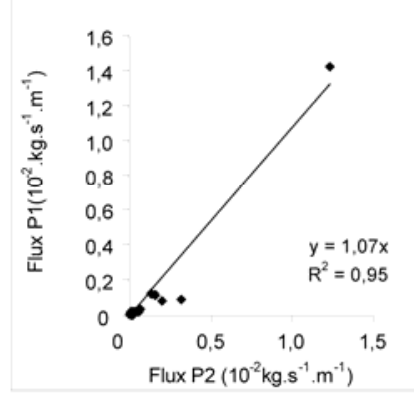

a)

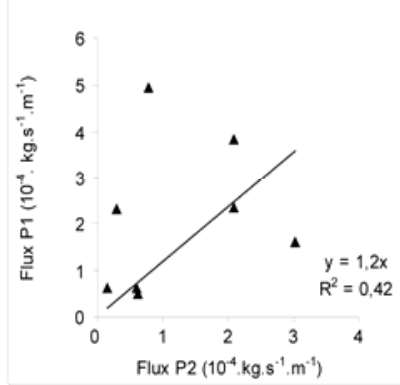

b)

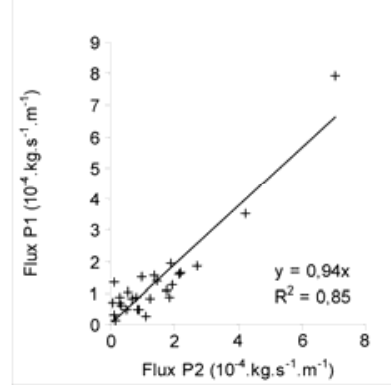

c)

Figure 4. Comparaison des flux sédimentaires entre les deux transects de mesures (P1 et P2) pour chaque site d'étude ( $a$ : Wissant ; $b$ : Zuydcoote ; $c$ : Hardelot).

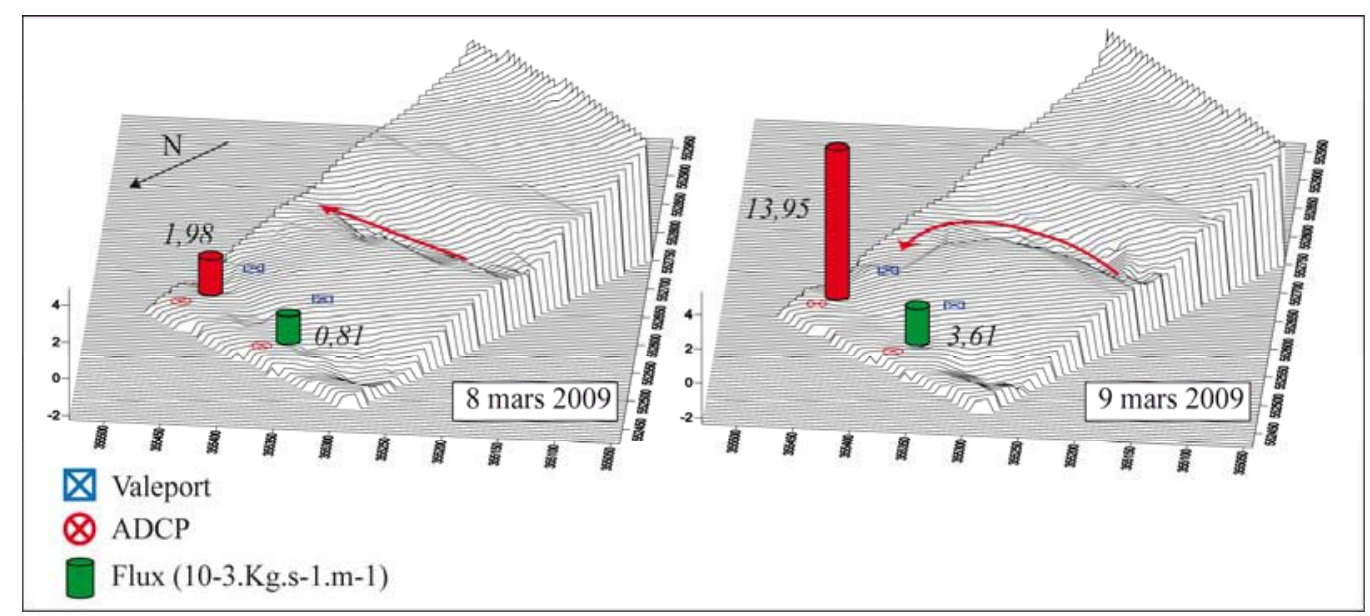

Figure 5. Influence de la morphologie de la plage sur les flux sédimentaire longshore, Wissant mars 2009.

\section{Conclusion}

Bien que nos analyses aient pu montrer que la variabilité des flux sédimentaires pouvait être mise en relation avec les variations de hauteur de houle et de vitesse moyenne du courant, il est difficile à ce stade de déterminer l'importance respective de chaque paramètre, car l'intensité du transport résulte de l'action combinée des vagues et des courants. Nos résultats montrent cependant que lorsqu'ils agissent seuls, les courants de marée s'avèrent peu efficaces pour induire un transport sédimentaire dans la zone intertidale, ce qui s'est produit lors de la campagne d'Hardelot, par exemple, où malgré un fort marnage les flux sédimentaires n'ont pas dépassé $0,18 \times 10^{-3} \mathrm{~kg} \mathrm{~s}^{-1} \mathrm{~m}^{-1}$. Les faibles valeurs de transport sédimentaire lors de cette campagne de mesures 
s'expliquent non seulement par une faible agitation de surface, mais aussi par de faibles courants de marée dont l'intensité diminue des petits fonds vers les plages (HEMDANE, 2006). L'action de la houle semble donc déterminante pour la mise en mouvement du sédiment, mais le rôle des courants de marée n'en est pas négligeable pour autant, car ils peuvent renforcer ou au contraire limiter le transport sédimentaire tout dépendant de leur direction par rapport au courant de dérive engendré par les vagues. Les résultats obtenus montrent une faible variabilité longitudinale des transports longshore sur les trois sites, mais aussi que la morphologie de barres et de bâches dans la zone intertidale peut induire localement une augmentation des flux sédimentaires à proximité de chenaux de vidanges de bâches, ce qui avait déjà été suggéré notamment par SIPKA \& ANTHONY (1999).

\section{Références}

ANTHONY E.J., HEQUETTE A. (2007). The grain size characterisation of coastal sand from the Somme estuary to Belgium: sediment segregation processes and sources consideration. Sedimentary Geology, 202, pp 369-382. doi:10.1016/j.sedgeo.2007.03.022

BAYRAM A., LARSON M., HANSON H. (2007). A new formula for the total longshore sediment transport rate. Coastal Engineering, 54, 9, pp 700-710. doi:10.1016/j.coastaleng.2007.04.001

HEMDANE Y. (2006). Dynamique hydro-sédimentaire d'une avant côte de type macrotidale : Le cas du littoral du Nord pas de Calais, France. Thèse de doctorat de Géograhie physique, Université du Littorale Côte d'Opale, 206 p.

KRAUS N.C. (1987). Application of portable traps for obtaining point measurements of sediment transport rates in the surf zone. Journal of Coastal Research, 3, pp 139-152.

LEVOY F., ANTHONY E.J., MONFORT O., LARSONNEUR C. (2000). The morphodynamics of megatidal beaches in Normandy, France. Marine Geology, 171, pp 39-59. doi:10.1016/S0025-3227(00)00110-9

ROSATI J.D., KRAUS N.C. (1989). Development of a portable sand trap for use in the nearshore. Department of the Army, U.S. Corps of Engineers. Technical report CERC, 89-91, $181 \mathrm{p}$.

SEDRATI M., ANTHONY E.J. (2007). Storm-generated morphological change and longshore sand transport in the intertidal zone of a multi-barred macrotidal beach. Marine Geology, 244, pp 209-229. doi:10.1016/j.margeo.2007.07.002

SIPKA V., ANTHONY E.J. (1999). Morphology and Hydrodynamics of a macrotidal ridge and runnel beach under modal low conditions. Journal de Recherche Océanographique, 24, pp 25-31.

VOULGARIS G., SIMONDS D., MICHEL D., HOWA H., COLLINS M.B., HUNTLEY D.A. (1998). Measuring and modelling sediment transport on a macrotidal ridge and runnel beach: an intercomparison. Journal of Coastal Research, 14, pp 315-330. 